\title{
Possible role of natural Italian populations of white clover for reclamation purposes in sub- humid and humid Mediterranean environ-
} ments (1)

Valeria NEGRI \& Fabio VERONESI

Istituto di Miglioramento Genetico Vegetale, Università degli Studi di Perugia, Italy environment need to be further verified.

Additional key words : Breeding, natural populations, seed import, Trifolium repens $L$., pastures, submediterranean environments, germplasm, agronomic evaluation.

Utilisation possible de populations naturelles italiennes du trèfle blanc pour les semis des pâturages dans l'environnement sub-méditerranéen.

RÉSUMÉ

\begin{abstract}
Pour commencer un programme de sélection variétale du trèfle blanc (Trifolium repens L.) pour l'Europe subméditerranéenne, 48 populations naturelles ont été collectées durant l'été 1981.

Ces populations et 3 variétés témoins néo-zélandaises disponibles sur le marché ont été évaluées en 1983/84 à Pérouse, Italie, pour la dormance hivernale, la repousse printanière, la date de floraison, les productions de matière sèche et de semences. Les résultats indiquent l'existence d'une large variabilité inter-populations et suggèrent que l'amélioration génétique peut aboutir.

On constate que quelques-uns de nos écotypes produisent plus que des variétés commerciales. Si ce résultat se maintenait en peuplement dense, une simple multiplication des meilleurs écotypes pourrait constituer la première étape de résolution des problèmes liés aux semis des pâturages et à l'importation de semences.

Les études relatives à la production des semences des écotypes (aptitude, techniques de production) ont besoin d'être approfondies.
\end{abstract}

Mots clés additionnels : Sélection, populations naturelles, importation de semences, Trifolium repens $L$., pâturages, environnement sub-méditerranéen, germoplasme, évaluation agronomique.

\section{INTRODUCTION}

The sub-humid and humid Mediterranean zones are very extensive in Portugal, Spain, France, Yugoslavia,

(1) Research supported by Ministero dell'Agricoltura e delle Foreste, special Projet "Foraggero Zootecnico". Paper presented at the EEC Workshop on Nutrition, Agronomy and Breeding of White Clover, Johnstown Castle Research Center, Wexford, Ireland, 2-4 July 1985 .
Italy, Greece and Turkey and also occupy large areas in the mountain regions of the Near East (Lebanon, Syria, Jordan, Palestine, Iraq, Iran). Although there are extreme ecological, historical and agricultural variations around the Mediterranean, the sub-humid Mediterranean zone can be defined climatically by an average annual rainfall between 600 and $800 \mathrm{~mm}$ and an Emberger index from 70 (cold winters) to 120 (warm winters). The humid Mediterranean zone can be defined by an average annual rainfall between 800 and 
$1200 \mathrm{~mm}$ and an Emberger index from 90 (cold winters) to 150 warm winters (LE HOUÉROU, 1977).

In the last two decades there has been increased interest in the use of legumes in these regions for several reasons : 1) to reclaim marginal lands abandoned after the rapid industrialization process that followed World War II by sowing pastures, 2) to reduce the need for costly nitrogen fertilizers and 3) to improve feed value and yield potential of the swards.

Little data are available on suitable species and cultivars to support a breeding project for these environments. In such a situation the first problem is the choice of species, those native to the area probably being the most suitable. A survey in pastures naturally established on hill lands which were once ploughed indicated that over 60 legume species were present. The most widely distributed were some good forages such as Medicago lupulina L., Lotus corniculatus L., Trifolium repens $\mathrm{L}$., Trifolium campestre $\mathrm{L}$. and, to a lesser extent, Onobrychis viciifolia Scop. (BENCIVENGA \& NEGRI, 1983). Therefore in starting a breeding program aimed at synthesizing varieties for these environments we did not feel the necessity to introduce exotic germplasm. Besides, the use of native materials avoids the risk of poor adaptation which could further complicate the breeding.

Since perennial legume species appear to be more important than annuals in this area, attention was focused on L. corniculatus, $T$. repens and $O$. vicilfolia. The first step was to make a germplasm collection of the species under investigation in order to assess the amount of variation and eventually find valuable populations with which to start a breeding program.

This paper synthesizes the results on the agronomic evaluation of a Central Italian white clover collection. Other data concerning a much wider collection from Central Europe, Mediterranean and others countries can be found in ELLIS DAVIS \& YOUNG (1967).

Results concerning birdsfoot trefoil and sainfoin have been presented elsewhere (VERONESI \& NEGRI, 1985 ; NEGRI et al., 1987).

\section{MATERIALS AND METHODS}

Forty-eight natural white clover populations were collected in Central Italy in 1981 in an area ranging from $44^{\circ} 10^{\prime}$ to $42^{\circ} 12^{\prime}$ latitude North and from $10^{\circ} 20^{\prime}$ to $13^{\circ} 33^{\prime}$ longitude East (Table 1). The procedure reported by Hawkes (1980) was utilized in making the germplasm collection. Altitudes, climatic and geographic characteristics and habitats relative to collection sites are also reported in Table 1 . In the paper natural populations will be referred to by their ass yned numisers.

Two hundred scarified seeds for each natural population and for each of three New Zealand cultivars used as controls, New Zealand dwarf, Ellet and Huia, were put in Petri dishes to germinate on February 26, 1982. New Zealand cultivars were chosen because most of the white clover variety sown in Europe is represented by cv. Huia.

Seedlings were pricked out in Jiffy pots and grown under greenhouse conditions. Sixty randomly chosen plants were transplanted and spaced $75 \times 75 \mathrm{~cm}$ apart in the experimental field at Collestrada, Perugia, on April 24, 1982. A randomized complete block design with three replications was used.

The following characters were recorded in 1983-84.

1) Winter dormancy measured twice on January 20 and February 23, 1983. Each plant was scored ( 1 = dormant ; 2 = showing different amounts of green foliage) and the populations were classified according to the percentages of dormancy (ratio between plants scored 1 and total number of plants $\times$ 100 ), values per replicate were calculated and then transformed in arcsin values ;

2) Spring regrowth scored on single plant basis on March 23 and April 11, 1983 (1 - poorest, 9 - best), average values per replicate were then calculated ;

3) Bloom date (days from May 1st, 1983) recorded for replication at each successive blooming when more than $90 \%$ of the plants showed at least 3 inflorescences with a floret that could be visited by bees ;

4) Dry matter yield (DMY) measured as g/plant on each of the four bloom dates in 1983 (which on the average occured on May 20, June 16, July 21 and October 11) ;

5) Seed production $\left(\mathrm{g} / \mathrm{m}^{2}\right.$ under open pollination conditions) recorded after a pre-cut in May, 1984 ; since stolons produced a continuous sward, two samples of one square meter each, per replicate, were taken. No irrigation was applied and pollination was fully provided by honey bees, 4-10 honey bees $/ \mathrm{m}^{2}$ always being present during the pollination period. This number is estimated to be sufficient to trip forage legume seed crops (FAVERO, 1980).

\section{RESULTS AND DISCUSSION}

Data are presented as frequency histograms relative to the bulk of populations. In the histograms positions relative to Huia and one of the most productive ecotypes (n. 32 Comunali di Rieti) are indicated by arrows, while averages relative to each one of them can be found in table 2. Analysis of variance results always showed significant differences among materials for all the examined characters, with the exception of the fourth bloom date.

Data relative to winter dormancy for the two sampling dates are reported in figure 1 and table 2.

Winter dormancy increased from January to February. On January 20, only one of the examined populations (n. $37 \overline{\mathrm{x}}=45.8$ ) showed a percentage of winter dormancy above $40.0 \%$ while on February 23, 16 of them were above this figure. On the second sampling date, 15 populations still appeated to be nondormant. At both sampling dates Huia seemed to be a non-dormant material, the other two control varieties differing from Huia only on the second sampling date.

Generally, the onset of winter dormancy falls around the end of January which in Central Italy is the coldest period of the year. Fopulations of $T$. repens appear able to remain in active vegetation for a longer period in late autumn than populations of Medicago sativa coming from the same area (LORENZETTI et al., 1972). This has already been observed for $L$. corniculatus (VERONESI \& NEGRI, 1985). 
TABLE 1

Altitude, rainfall, exposure, longitude, latitude and habitat relative to collection sites. Altitude, pluviosité, exposition, longitude, latitude et habitat des zones de collecte.

\begin{tabular}{|c|c|c|c|c|c|c|}
\hline Collection sites & $\begin{array}{c}\text { Altitude } \\
\text { ma.s.l. }\end{array}$ & $\begin{array}{l}\text { Rainfall } \\
\text { mm }\end{array}$ & Exposure & Longitude & Latitude & Habitat \\
\hline 1) Cesenatico (Forli) & 5 & 730 & open & $12^{\circ} 22^{\prime}$ & $44^{\circ} 10^{\prime}$ & wasteland, along sea \\
\hline 2) Roseto degli Abruzzi (Teramo) & 8 & 693 & open & $14^{\circ} 02^{\prime}$ & $42^{\circ} 38^{\prime}$ & wasteland, along sea \\
\hline 3) Pescara & 8 & 780 & open & $14^{\circ} 12^{\prime}$ & $42^{\circ} 29^{\prime}$ & wasteland, along sea \\
\hline 4) Tarquinia (Viterbo) & 8 & 638 & open & $11^{\circ} 43^{\prime}$ & $42^{\circ} 12^{\prime}$ & wasteland, edgerow \\
\hline 5) Scarlino (Grosseto) & 10 & - & not recorded & $10^{\circ} 58^{\prime}$ & $42^{\circ} 58^{\prime}$ & wasteland, along sea \\
\hline 6) Giulianova (Teramo) & 20 & 615 & not recorded & $13^{\circ} 58^{\prime}$ & $42^{\circ} 45^{\prime}$ & wasteland, edgerow \\
\hline 7) Torre Trappola (Grosseto) & 20 & 549 & open & $11^{\circ} 03^{\prime}$ & $42^{\circ} 42^{\prime}$ & grassland \\
\hline 8) Marina di Montalto di Castro (Viterbo) & 30 & 638 & SW & $11^{\circ} 41^{\prime}$ & $42^{\circ} 16^{\prime}$ & wasteland grazed \\
\hline 9) Lago di Massaciuccoli (Lucca) & 50 & 1154 & open & $10^{\circ} 20^{\prime}$ & $43^{\circ} 50^{\prime}$ & grassland, clearing \\
\hline 10) Rosignano (Livorno) & 50 & 807 & W & $10^{\circ} 26^{\prime}$ & $43^{\circ} 18^{\prime}$ & arable, hedgerow \\
\hline 11) Cecina (Livorno) & 50 & 807 & open & $11^{\circ} 25^{\prime}$ & $43^{\circ} 17^{\prime}$ & abandoned meadow \\
\hline 12) Donoratico (Livorno) & 50 & 710 & open & $10^{\circ} 43^{\prime}$ & $43^{\circ} 10^{\prime}$ & wasteland grazed \\
\hline 13) Pesaro & 90 & 815 & open & $12^{\circ} 57^{\prime}$ & $43^{\circ} 55^{\prime}$ & backyard \\
\hline 14) Sambucheto (Macerata) & 150 & 758 & open & $13^{\circ} 21^{\prime}$ & $43^{\circ} 21^{\prime}$ & wasteland grazed \\
\hline 15) Piano di Torreorsina (Terni) & 150 & 999 & open & $12^{\circ} 47^{\prime}$ & $42^{\circ} 31^{\prime}$ & arable, hedgerow \\
\hline 16) Mercato Saraceno (Forli) & 160 & 1000 & $\mathrm{~N}$ & $12^{\circ} 10^{\prime}$ & $43^{\circ} 58^{\prime}$ & wasteland \\
\hline 17) Ascoli Piceno & 190 & 772 & open & $13^{\circ} 33^{\prime}$ & $42^{\circ} 49^{\prime}$ & wasteland, along river \\
\hline 18) Sarsina (Forli) & 220 & 1053 & $\mathrm{~N}$ & $12^{\circ} 09^{\prime}$ & $43^{\circ} 54^{\prime}$ & wasteland \\
\hline 19) Collestrada (Perugia) & 247 & 773 & open & $12^{\circ} 30^{\prime}$ & $43^{\circ} 08^{\prime}$ & grassland \\
\hline 20) Poggiarello (Siena) & 250 & 863 & SW & $11^{\circ} 21^{\prime}$ & $43^{\circ} 20^{\prime}$ & wasteland \\
\hline 21) Str. Pitigliano (Grosseto) & 250 & 922 & open & $11^{\circ} 42^{\prime}$ & $42^{\circ} 35^{\prime}$ & arable, hedgerow \\
\hline 22) Saturnia Terme (Grosseto) & 250 & 922 & NW & $11^{\circ} 19^{\prime}$ & $42^{\circ} 30^{\prime}$ & wasteland \\
\hline 23) Panicarola (Perugia) & 275 & 1006 & open & $12^{\circ} 04^{\prime}$ & $43^{\circ} 06^{\prime}$ & wasteland, along road \\
\hline 24) Macerata & 290 & 782 & W & $13^{\circ} 25^{\prime}$ & $43^{\circ} 18^{\prime}$ & wasteland \\
\hline 25) Querce al Pino (Siena) & 300 & 716 & W & $11^{\circ} 55^{\prime}$ & $43^{\circ} 00^{\prime}$ & meadow \\
\hline 26) Monte Argentario (Grosseto) & 300 & - & $\mathrm{NE}$ & $11^{\circ} 10^{\prime}$ & $42^{\circ} 22^{\prime}$ & Mediterranean forest \\
\hline 27) Todi (Perugia) & 320 & 885 & $\mathbf{E}$ & $12^{\circ} 25^{\prime}$ & $42^{\circ} 45^{\prime}$ & wasteland \\
\hline 28) Agello (Perugia) & 350 & 874 & open & $12^{\circ} 18^{\prime}$ & $43^{\circ} 05^{\prime}$ & wasteland \\
\hline 29) Pieve d'Agnano (Perugia) & 370 & 729 & NW & $12^{\circ} 21^{\prime}$ & $43^{\circ} 15^{\prime}$ & grassland, along forest \\
\hline 30) Piegaro (Perugia) & 400 & 941 & $\mathrm{~N}$ & $12^{\circ} 01^{\prime}$ & $42^{\circ} 58^{\prime}$ & wasteland \\
\hline 31) Rieti & 400 & 1180 & open & $12^{\circ} 51^{\prime}$ & $42^{\circ} 23^{\prime}$ & wasteland \\
\hline 32) Comunali (Rieti) & 400 & 1180 & open & $12^{\circ} 51^{\prime}$ & $42^{\circ} 22^{\prime}$ & arable, hedgerow \\
\hline 33) Polcanto (Firenze) & 450 & 1078 & $N$ & $11^{\circ} 21^{\prime}$ & $43^{\circ} 54^{\prime}$ & grassland \\
\hline 34) Canetra (Rieti) & 450 & 1180 & open & $13^{\circ} 00^{\prime}$ & $42^{\circ} 25^{\prime}$ & wasteland, along river \\
\hline 35) Torre a Buiano (Firenze) & 460 & 928 & $\mathrm{~S}$ & $11^{\circ} 20^{\prime}$ & $43^{\circ} 47^{\prime}$ & wasteland, along road \\
\hline 36) Montefranco (Terni) & 500 & - & W & $12^{\circ} 49^{\prime}$ & $42^{\circ} 40^{\prime}$ & wasteland \\
\hline 37) Gubbio (Perugia) & 600 & 1037 & SW & $12^{\circ} 33^{\prime}$ & $43^{\circ} 22^{\prime}$ & wasteland \\
\hline 38) Sarteano (Siena) & 600 & - & $\mathbf{E}$ & $11^{\circ} 59^{\prime}$ & $43^{\circ} 00^{\prime}$ & wasteland \\
\hline 39) Visso (Macerata) & 620 & 993 & open & $13^{\circ} 04^{\prime}$ & $42^{\circ} 56^{\prime}$ & wasteland, along ditch \\
\hline 40) Tuoro (Perugia) & 650 & 765 & $\mathrm{SE}$ & $12^{\circ} 05^{\prime}$ & $43^{\circ} 05^{\prime}$ & old meadow \\
\hline 41) S. Paterniano (Perugia) & 700 & 1018 & $\mathbf{E}$ & $13^{\circ} 05^{\prime}$ & $42^{\circ} 58^{\prime}$ & wasteland, along road \\
\hline 42) Colfiorito (Perugia) & 760 & 1252 & open & $12^{\circ} 50^{\prime}$ & $42^{\circ} 59^{\prime}$ & grassland \\
\hline 43) Norcia (Perugia) & 830 & 800 & W & $13^{\circ} 04^{\prime}$ & $42^{\circ} 47^{\prime}$ & forest clearing \\
\hline 44) Abbadia S. Salvatore (Siena) & 350 & 1401 & not recorded & $11^{\circ} 45^{\prime}$ & $42^{\circ} 55^{\prime}$ & forest clearing \\
\hline 45) Monte Subasio 1040 (Perugia) & 1040 & - & $\mathbf{E}$ & $12^{\circ} 40^{\prime}$ & $43^{\circ} 05^{\prime}$ & grassland \\
\hline 46) Valico delle Capannelle (L'Aquila) & 1260 & 1036 & $\mathrm{~N}$ & $13^{\circ} 23^{\prime}$ & $42^{\circ} 23^{\prime}$ & grassland \\
\hline 47) Monte Subasio 1270 (Perugia) & 1270 & - & $\mathbf{E}$ & $12^{\circ} 40^{\prime}$ & $43^{\circ} 05^{\prime}$ & grassland \\
\hline 48) Leonessa (Rieti) & 1600 & 1503 & not recorded & $12^{\circ} 59^{\prime}$ & $42^{\circ} 33^{\prime}$ & grassland \\
\hline
\end{tabular}

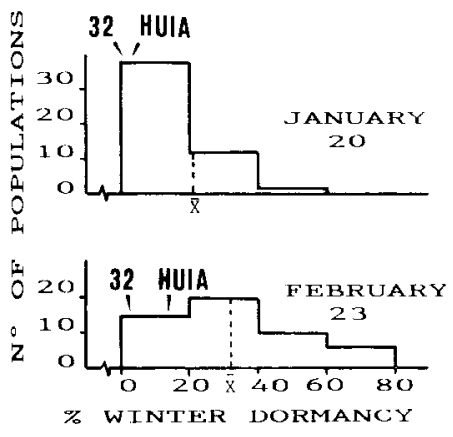

Figure 1

Percentage of winter dormancy (on January 20 and February 23) histograms relative to the examined populations.

Histogrammes des pourcentages de dormance hivernale rau 20 janvier et 23 février) des populations étudiées.
Data relative to spring regrowth (fig. 2 and table 2) showed an increase in vegetative activity from March $23(\bar{x}=4.0)$ to April $11(\mathrm{x}=5.5)$. A wide range of variability was found for this character on both sampling dates. For example on March 23, 7 populations (n. $4,8,13,16,26,32,42$ ) already showed good spring regrowth, while 5 populations (n. 29, 37, 41, 46, 47) were still dormant. The control variety Huia showed active spring regrowth on both sampling dates. The other two control varieties did not differ from Huia for this character as well as for the following ones and consequently will not be mentioned further.

As for bloom dates, all populations flowered, on the average, four times in mid-May, mid-June, mid-July and the beginning of October in 1983, with the 
TABLE 2

Winter dormancy percentages on January 20 and February 23, 1983 (arcsin transformation was used for statistical analysis, data are presented in actual units), average spring regrowth $(1=$ poor, $9=$ best) on March 23 and April 11, 1983, first full bloom date (days from May 1st 1983), annual DMY (1983, $\mathrm{g} /$ plant) and 1984 seed yield $\left(\mathrm{g} / \mathrm{m}^{2}\right)$ relative to the examined populations.

Pourcentages de dormance hivernale au 20 janvier et au 23 février 1983 (la transformation en arcsinus a été utilisée pour l'analyse statistique ; les données sont présentées en valeurs réelles), la repousse printanière $(1=$ très faible, $9=$ très importante $)$ au 23 mars et au 11 avril 1983, date de la première floraison (en jours à partir du $\mathrm{I}^{\text {er }}$ mai), la production totale de matière sèche (en $1983, \mathrm{~g} / \mathrm{plante}$ ) et la production de semences $\left(\mathrm{g} / \mathrm{m}^{2}\right)$ des populations étudiées.

\begin{tabular}{|c|c|c|c|c|c|c|c|}
\hline \multirow[t]{2}{*}{ Collection sites } & \multicolumn{2}{|c|}{$\begin{array}{c}\text { Winter } \\
\text { dormancy } \%\end{array}$} & \multicolumn{2}{|c|}{$\begin{array}{l}\text { Spring } \\
\text { regrowth }\end{array}$} & \multirow{2}{*}{$\begin{array}{c}\begin{array}{c}\text { 1st bloom } \\
\text { date }\end{array} \\
\begin{array}{c}\text { dd. from } \\
\text { May } 1\end{array}\end{array}$} & \multirow{2}{*}{$\begin{array}{c}\begin{array}{c}\text { Annual } \\
\text { DMY }\end{array} \\
\text { g/plant }\end{array}$} & \multirow{2}{*}{$\begin{array}{r}\begin{array}{r}\text { Seed } \\
\text { yield }\end{array} \\
\mathrm{g} / \mathrm{m}^{2}\end{array}$} \\
\hline & Jan. 20 & Feb. 23 & $\begin{array}{l}\text { March } \\
23\end{array}$ & $\begin{array}{l}\text { April } \\
11\end{array}$ & & & \\
\hline 1) Cesenatico (Forli) & $34.1 \mathrm{sqr}^{*}$ & $52.5 \mathrm{vw}$ & 3.9 & 5.3 & 16 & 138.4 & 23.5 \\
\hline 2) Roseto degli Abruzzi (Teramo) & 10.9 ghijk & $36.3 \mathrm{opq}$ & 4.5 & 5.3 & 26 & 119.2 & 13.4 \\
\hline 3) Pescara & $0.1 \mathrm{a}$ & $17.8 \mathrm{efgh}$ & 4.9 & 6.5 & 16 & 79.1 & - \\
\hline 4) Tarquinia (Viterbo) & $4.3 \mathrm{abc}$ & $8.7 \mathrm{abcd}$ & 5.6 & 5.9 & 27 & 107.5 & 18.4 \\
\hline 5) Scarlino (Grosseto) & $14.8 \mathrm{jx} \operatorname{lmn}$ & 31.4 mnop & 3.7 & 4.9 & 15 & 90.9 & 8.4 \\
\hline 6) Giulianova (Teramo) & $13.0 \mathrm{ijxlm}$ & $37.0 \mathrm{opqr}$ & 3.7 & 5.2 & 9 & 91.7 & 9.3 \\
\hline 7) Torre Trappola (Grosseto) & 10.4 fgnij & $43.7 \mathrm{rstu}$ & 3.8 & 5.3 & 17 & 98.4 & 11.9 \\
\hline 8) Marina di Montalto di Castro (Vt)) & $0.0 \mathrm{a}$ & $5.9 \mathrm{ab}$ & 5.9 & 7.2 & 27 & 172.4 & 15.0 \\
\hline 9) Lago di Massaciuccoli (Lucca) & $15.9 \mathrm{lmn}$ & $35.6 \mathrm{nopq}$ & 3.8 & 5.2 & 25 & 159.1 & 8.1 \\
\hline 10) Rosignano (Livorno) & $25.0 \mathrm{p}$ & $25.0 \mathrm{nijklm}$ & 3.7 & 6.0 & 17 & 256.7 & $\ldots$ \\
\hline 11) Cecina (Livorno) & $13.7 \mathrm{ijklm}$ & 35.3 nopq & 3.8 & 5.2 & 17 & 102.4 & 6.8 \\
\hline 12) Donoratico (Livorno) & $38.7 \mathrm{t}$ & 58.1 wxy & 3.3 & 4.6 & 16 & 66.7 & 7.3 \\
\hline 13) Pesaro & $12.2 \mathrm{hijkl}$ & 21.9 ghijk & 5.3 & 6.3 & 16 & 99.2 & 17.7 \\
\hline 14) Sambucheto (Macerata) & $16.9 \mathrm{mn}$ & 38.5 pqrs & 3.2 & 4.7 & 16 & 96.6 & 19.2 \\
\hline 15) Piano di Torre Orsina (Terni) & 7.7 cdefgh & $30.7 \mathrm{Imno}$ & 4.7 & 6.4 & 27 & 144.1 & 21.9 \\
\hline 16) Mercato Saraceno (Forli) & $1.5 \mathrm{ab}$ & $8.8 \mathrm{abcd}$ & 5.5 & 6.5 & 9 & 133.4 & 23.4 \\
\hline 17) Ascoli Piceno & 10.0 efghi & 16.0 defg & 3.9 & 5.2 & 16 & 110.9 & 9.2 \\
\hline 18) Sarsina (Forli) & 9.7 efghi & 35.5 nopq & 4.9 & 6.1 & 16 & 175.0 & - \\
\hline 19) Collestrada (Perugia) & 22.2 nop & $47.6 \mathrm{uv}$ & 3.0 & 5.0 & 17 & 98.3 & 16.8 \\
\hline 20) Poggiarello (Siena) & $24.5 \mathrm{p}$ & 40.0 qrsi & 3.9 & 5.5 & 26 & 167.6 & 13.0 \\
\hline 21) Str. Pitigliano (Grosseto) & 6.4 cdefg & $63.8 \mathrm{yz}$ & 3.3 & 4.8 & 17 & 76.6 & 7.9 \\
\hline 22) Saturnia Terme (Grosseto) & 5.7 bcde & $8.6 \mathrm{abc}$ & 4.8 & 6.2 & 16 & 75.0 & - \\
\hline 23) Panicarola (Perugia) & $19.0 \mathrm{mno}$ & 46.4 tuv & 3.5 & 5.0 & 23 & 105.7 & 6.9 \\
\hline 24) Macerata & $4.6 \mathrm{bc}$ & 11.6 bcde & 4.6 & 6.2 & 16 & 138.3 & 9.1 \\
\hline 25) Querce al Pino (Siena) & $15.1 \mathrm{k} \operatorname{lmn}$ & 30.3 lmno & 3.7 & 4.8 & 23 & 158.2 & 5.5 \\
\hline 26) Monte Argentario (Grosseto) & $3.2 \mathrm{abc}$ & $3.2 \mathrm{a}$ & 6.2 & 7.3 & 17 & 85.0 & 7.8 \\
\hline 27) Todi (Perugia) & $25.0 \mathrm{p}$ & $62.5 \mathrm{xyz}$ & 3.5 & 4.8 & 17 & 92.5 & 12.4 \\
\hline 28) Agello (Perugia) & 9.3 defghi & 20.4 fghij & 3.8 & 5.4 & 23 & 171.7 & 10.9 \\
\hline 29) Pieve d'Agnano (Perugia) & $29.8 \mathrm{q}$ & $36.8 \mathrm{opq}$ & 2.9 & 4.8 & 23 & 75.8 & 11.7 \\
\hline 30) Piegaro (Perugia) & 5.9 bcdef & 41.1 qrstu & 3.3 & 4.9 & 23 & 140.0 & 7.3 \\
\hline 31) Rieti & $0.0 \mathrm{a}$ & 9.8 abcd & 4.7 & 6.3 & 27 & 140.0 & 13.5 \\
\hline 32) Comunali (Rieti) & $0.0 \mathrm{a}$ & $2.8 \mathrm{a}$ & 5.3 & 6.8 & 27 & 190.0 & - \\
\hline 33) Polcanto (Firenze) & $36.2 \mathrm{rs}$ & $61.7 \mathrm{xyz}$ & 3.9 & 5.0 & 17 & 122.5 & 16.3 \\
\hline 34) Canetra (Rieti) & $1.4 \mathrm{ab}$ & 20.0 fghi & 3.9 & 5.8 & 27 & 144.1 & 11.0 \\
\hline 35) Torre a Buiano (Firenze) & $25.0 \mathrm{p}$ & 38.9 qrs & 4.0 & 6.1 & 25 & 146.7 & 10.2 \\
\hline 36) Montefranco (Terni) & $5.0 \mathrm{bcd}$ & $45.0 \mathrm{stu}$ & 4.2 & 5.6 & 17 & 88.3 & 12.4 \\
\hline 37) Gubbio (Perugia) & $45.8 \mathrm{t}$ & $66.1 \mathrm{z}$ & 2.7 & 4.2 & 17 & 99.2 & 14.7 \\
\hline 38) Sarteano (Siena) & $24.1 \mathrm{p}$ & $27.5 \mathrm{jklm}$ & 4.0 & 5.3 & 23 & 105.1 & 4.8 \\
\hline 39) Visso (Macerata) & 6.9 cdefg & 11.6 bcde & 4.5 & 6.1 & 17 & 115.8 & 16.0 \\
\hline 40) Tuoro (Perugia) & $35.5 \mathrm{rs}$ & $56.4 \mathrm{wx}$ & 3.0 & 4.5 & 23 & 83.3 & 5.8 \\
\hline 41) S. Paterniano (Perugia) & 10.0 efghi & $63.3 \mathrm{xyz}$ & 2.9 & 4.8 & 17 & 75.0 & 10.0 \\
\hline 42) Colfiorito (Perugia) & 10.5 ghij & $23.6 \mathrm{hijkl}$ & 5.3 & 6.8 & 23 & 194.2 & - \\
\hline 43) Norcia (Perugia) & 7.4 cdejg & $25.9 \mathrm{ijklm}$ & 3.7 & 5.4 & 17 & 86.0 & 9.0 \\
\hline 44) Abbadia S. Salvatore (Siena) & 9.5 defghi & $28.5 \mathrm{k} \operatorname{lmn}$ & 3.4 & 5.0 & 17 & 90.0 & 10.3 \\
\hline 45) Monte Subasio 1040 (Perugia) & $16.6 \mathrm{Imn}$ & $48.2 \mathrm{uv}$ & 3.2 & 4.6 & 26 & 78.3 & 8.8 \\
\hline 46) Valico delle Capannelle (Aq) & 5.6 bcde & 47.2 tuv & 2.8 & 4.2 & 27 & 204.2 & - \\
\hline 47) Monte Subasio 1270 (Perugia) & 23.4 nop & $61.5 \mathrm{xyz}$ & 2.9 & 4.3 & 23 & 81.6 & 11.0 \\
\hline 48) Leonessa (Rieti) & $0.0 \mathrm{a}$ & 37.1 opqr & 3.1 & 4.2 & 27 & 139.2 & 19.1 \\
\hline 49) New Zealand dwarf & $0.0 \mathrm{a}$ & $8.6 \mathrm{abc}$ & 4.6 & 6.2 & 17 & 88.3 & 11.0 \\
\hline 50) Ellet & $1.6 \mathrm{ab}$ & $6.6 \mathrm{ab}$ & 4.4 & 5.6 & 17 & 75.0 & 9.6 \\
\hline 51) Huia & $3.2 \mathrm{abc}$ & $14.5 \mathrm{cde}$ & 4.7 & 5.9 & 17 & 78.4 & 12.3 \\
\hline$\overline{\mathrm{x}}$ & 21.0 & 32.0 & 4.0 & 5.5 & 20 & 120.0 & 11.5 \\
\hline $\mathrm{LSD}_{0.05}$ & - & - & 0.6 & 0.7 & 14 & 60.3 & 6.6 \\
\hline
\end{tabular}

* Means followed by the same letters are not significantly different per $\mathrm{P} \leqslant 0.05$.

exception of populations n. 3, 15 and 48 which only blossomed 3 times.

The first bloom ranged from May 9 to May 27 (fig. 3 and table 2). The earliest populations were n. 6 and n. 16 and the latest were n. $4,8,15,31,32,34,46$ and $48 ; \mathrm{cv}$. Huia flowered on May 17.

Only data relative to annual $D M Y$ per plant are reported in fig. 3 and table 2 , even if significant 


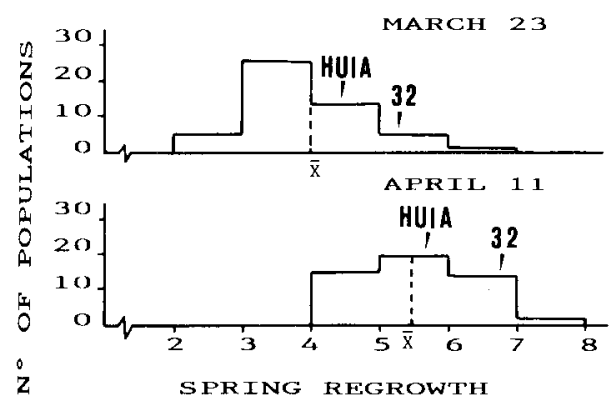

Figure 2

Spring regrowth (on March 23 April 11) histograms relative to the examined populations.

Histogrammes des pourcentages de repousse printanière (au 23 mars et 11 avril) des populations étudiées.

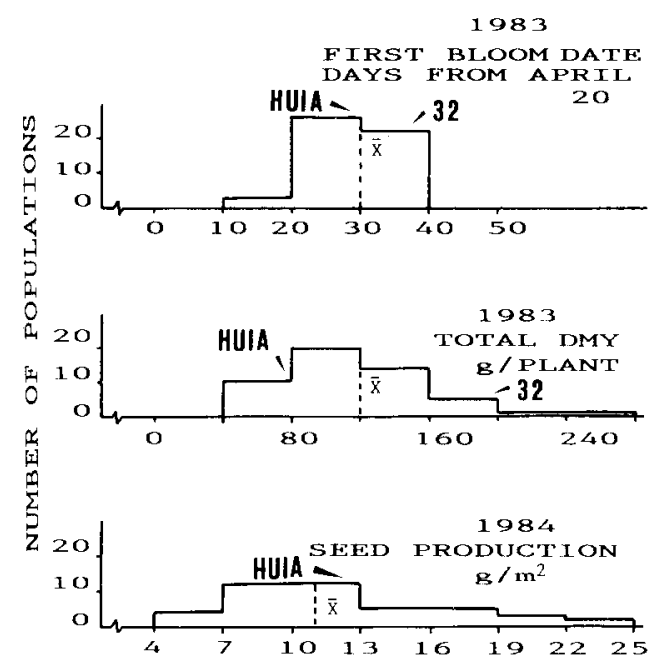

Figure 3

First bloom date (days from May 1) and annual DMY (g/plant) 1983 and seed yield $\left(\mathrm{g} / \mathrm{m}^{2}\right)$ in 1984 frequency histograms relative to the examined populations.

Histogrammes des dates de première floraison (en jours à partir du $l^{\text {er }}$ mai), de la production annuelle de matière sèche (g/plante) en 1983 et de la production de semences $\left(\mathrm{g} / \mathrm{m}^{2}\right)$ en 1984 des populations étudiées.

differences among populations were found for the first, second, third and fourth cuts. The range of variation for this character was very wide: annual DMY ranged from 66.7 (n. 12) to $256.7 \mathrm{~g} /$ plant (n. 10), with a general mean of $120.0 \mathrm{~g} /$ plant. Other outstanding natural populations were n. 32, 42 and 46 with over $185.0 \mathrm{~g} /$ plant. Surprisingly the plants of $\mathrm{cv}$. Huia gave very poor yields $(\mathrm{x}=78.4)$.

As far as seasonal growth pattern is concerned, on the average $60 \%$ of annual DMY was obtained from the first spring cut (May), $25 \%$ from the second and third cuts (June, July) and $15 \%$ from the autumn cut (October). It is noted that in respect to summer yielding ability population $\mathrm{n} .10$ produced $37.4 \%$ of its yield in June-July.

On the whole, populations n. 32 and 42 showing limited winter dormancy, early spring regrowth and high DMY could be regarded as valuable populations. Both populations n. 10 and 46, though more winter dormant and later in spring regrowth, also deserve some attention for their yielding ability.

Data relative to seed production under open pollination conditions in 1984 are reported in fig. 3 and table 2. Populations n. 32, 46, 10, 18 and 46 are not included because during this period they were submitted to multiplication in isolation cages with small honey-bee hives. Data relative to populations n. 3 and 22 were not recorded.

A wide variability was also found for this character. Seed production ranged from 5.5 (n. 25) to $23.5 \mathrm{~g} / \mathrm{m}^{2}$ (n. 1). Valuable ecotypes are also n. 15 and n. 16 ; cv. Huia gave a seed production of $12.3 \mathrm{~g} / \mathrm{m}^{2}$.

Assuming a $200-300 \mathrm{~kg} / \mathrm{ha}$ white clover seed yield and grazing of the seed crop from September to April, the net income from growing white clover for seed could be about as profitable as growing wheat in Central Italy. Further studies are needed to assess the seed yield potentialities of examined populations in appropriate trials when the management suggested by VERONESI \& LORENZETTI (1984) is applied. The use of irrigation on white clover grown for seed also needs to be investigated.

Finally, table 3 shows the correlation coefficients relative to the agronomic traits of the populations and the altitude of collection sites. Only correlations with data collected on February 23 for winter dormancy and on April 11 for spring regrowth are presented since at these dates the above-mentioned characters showed their peak manifestation. Correlations with data of January 20 and March 23 were not different from the above data (except for coefficient of correlations between spring regrowth on March 23 and total DMY, $\mathrm{r}=0.189$ n.s.).

Populations coming from higher altitudes showed a low degree of spring regrowth $\left(\mathrm{r}=-0.401^{*}\right)$ and later bloom date $\left(r=0.338^{*}\right)$. Spring regrowth

TABLE 3

Correlation coefficients relative to agronomic traits and altitude of collection sites of examined populations.

Coefficients de corrélation relatifs aux caractères agronomiques et à l'altitude des zones de collecte des populations étudiées.

\begin{tabular}{|c|c|c|c|c|c|}
\hline & $\begin{array}{l}\text { Altitude of } \\
\text { collection } \\
\text { sites }\end{array}$ & $\begin{array}{l}\text { Winter } \\
\text { dormancy } \\
\text { (Feb. 23) }\end{array}$ & $\begin{array}{l}\text { Spring } \\
\text { regrowth } \\
\text { (Apr. 11) }\end{array}$ & $\begin{array}{c}\text { 1st } \\
\text { Bloom } \\
\text { date }\end{array}$ & $\begin{array}{c}1983 \\
\text { Total DMY } \\
\text { g/plant }\end{array}$ \\
\hline Winter dormancy (Feb. 23) & 0.237 & - & & & \\
\hline Spring regrowth (Apr. 11) & $-0.401^{* *}$ & $-0.798^{* *}$ & - & & \\
\hline 1st Bloom date & $0.338^{*}$ & -0.033 & -0.008 & - & \\
\hline 1983 Total DMY & -0.002 & -0.191 & $0.298^{*}$ & $0.404^{* *}$ & - \\
\hline 1984 Seed yield & -0.081 & -0.102 & 0.247 & -0.057 & 0.258 \\
\hline
\end{tabular}

${ }^{*}=$ significant at $\mathrm{P} \leqslant 0.05$ level, ${ }^{* *}=$ significant at $\mathrm{P} \leqslant 0.01$ level. 
appeared to be negatively correlated with winter dormancy $\left(\mathrm{r}=-0.798^{* *}\right)$ and positively correlated with total DMY $\left(r=0.298^{*}\right)$ though this last coefficient appeared of scarce statistic relevance. Bloom date was also shown to positively affect total DMY $\left(r=0.404^{* *}\right)$ late populations being the most productive.

The most relevant finding appeared to be the lack of correlation between total DMY and seed yield suggesting that breeding for varieties characterized by both high dry matter and seed yield could be possible.

\section{CONCLUSIONS}

The wide variability among natural white clover populations for agronomic characters such as winter dormancy, spring regrowth, bloom dates, DMY and seed yield suggests that progress can be achieved in breeding programs aimed at synthesizing varieties to be used both for hay and pasture in sub-humid and humid Mediterranean Europe. This study is limited, however, to the extent that it only included data from one year, from one location and collected under spaced plant conditions.

In particular, some materials characterized by limited winter dormancy, early spring regrowth and good DMY could be used to attenuate the winter gap typical of this region. The natural populations $n .32$, 42,10 and 46 appear to be noteworthy in this respect. Among them, n. 10 also showed a relatively high production in July.

Since the most used cultivar in the region, Huia, as well as the other two tested in this experiment, does not appear able to compete with the natural populations for both DMY and seed yield, simple multiplication of the best ecotypes could be the first step in solving problems connected with sowing pastures and importing seed.

This will be checked in the future when, after multiplication in isolation cages, the best populations will be tested under dense stand conditions with the control variety Huia.

Reçu le 5 septembre 1986. Accepté le 5 juin 1987.

\section{AKNOWLEDGEMENTS}

The Authors wish to thank Prof. Franco LoREnzetTI, Director of Istituto di Miglioramento Genetico Vegetale, University of Perugia (Italy) and Prof. Paul MANSAT, I.N.R.A., Montpellier (France) for helpful discussions and advice. Thank are due also to Dr. Mary Traynor, University of Perugia, for her help with the English form of the paper and to Mr. Renzo TORRICEI.I.I for technical assistance.

\section{REFERENCES}

Bencivenga M., Negri V., 1983. Le leguminose dei pascoli umbri. Riv. di Agron., 17, 2 : 315-326.

Ellis Davies W., Young N. R., 1967. The characteristics of European, Mediterranean and other populations of white clover (Trifolium repens L.). Euphytica, $16: 330-340$.

Favero A., 1980. Sementi : normativa e produzione. Italia Agricola, $117: 20-133$.

Hawkes J. G., 1980. Crop Genetic Resources Field Collection Manual. IBPGR ana EUCARPIA, $37 \mathrm{p}$.

Le Houérou H. N., 1977. Plant sociology and ecology applied to grazing lands research, survey and management in the Mediterranean basin. In "Application of vegetation science to grassland husbandry", part XIII, Krause ed., Dr. W. Junk b.v. Publish., The Hague, 211-274.
Lorenzetti F., Ceccarelli S., Catena Q., 1972. Caratterizzazione di ecotipi e selezione in erba medica. Sementi Elette, $3: 17-28$.

Negri V., Veronesi F., Falcinelli M., 1987. Germplasm evaluation of Onobrychis viciifolia Scop. for agronomic traits. Genet. Agr., 41 : 25-40.

Veronesi F., Lorenzetti F., 1984. Agronomic prospects and limitations to white clover seed production in arid areas. Abstract of paper presented at the EEC Plant Productivity Group Workshop "White clover seed production"' Welsh Plant Breeding Station, Aberystwyth Nov. 13-15, 1984, Great Britain, 60-61.

Veronesi F., Negri V., 1985. Variability for agronomic characters among Lotus corniculatus L. natural populations collected in Central Italy. Genet. Agr., $39: 431-444$. 